\title{
DAP kinase regulates JNK signaling by binding and activating protein kinase $D$ under oxidative stress
}

\author{
A Eisenberg-Lerner ${ }^{1}$ and A Kimchi ${ }^{*, 1}$
}

\begin{abstract}
The stress-activated kinase JNK mediates key cellular responses to oxidative stress. Here we show that DAP kinase (DAPk), a cell death promoting Ser/Thr protein kinase, plays a main role in oxidative stress-induced JNK signaling. We identify protein kinase D (PKD) as a novel substrate of DAPk and demonstrate that DAPk physically interacts with PKD in response to oxidative stress. We further show that DAPk activates PKD in cells and that induction of JNK phosphorylation by ectopically expressed DAPk can be attenuated by knocking down PKD expression or by inhibiting its catalytic activity. Moreover, knockdown of DAPk expression caused a marked reduction in JNK activation under oxidative stress, indicating that DAPk is indispensable for the activation of JNK signaling under these conditions. Finally, DAPk is shown to be required for cell death under oxidative stress in a process that displays the characteristics of caspase-independent necrotic cell death. Taken together, these findings establish a major role for DAPk and its specific interaction with PKD in regulating the JNK signaling network under oxidative stress. Cell Death and Differentiation (2007) 14, 1908-1915; doi:10.1038/sj.cdd.4402212; published online 17 August 2007
\end{abstract}

DAP kinase (DAPk), a serine/threonine protein kinase, was identified in an unbiased genetic screen aimed at finding positive mediators of cell death. ${ }^{1}$ It has since been characterized as a tumor suppressor protein whose expression is greatly reduced or lost in a significant number of human malignancies. ${ }^{2,3}$ DAPk belongs to the family of $\mathrm{Ca}^{2+}-\mathrm{CAM}$ regulated kinases and contains a $\mathrm{Ca}^{2+}-\mathrm{CAM}$ binding domain proximal to the catalytic domain. The multidomain structure of DAPk also includes a stretch of ankyrin repeats, a cytoskeleton binding domain and a death domain shown to be required in some of the DAPk-induced cell death scenarios. ${ }^{3}$ The tumor-suppressive properties of DAPk can be associated with its role in eliciting cell death, as numerous cell death triggering signals require DAPk. ${ }^{3}$ Interestingly, various modes of programmed cell death have been linked to DAPk, each representing a different functional arm in the mode of action of this kinase, depending on the type of cells and the specific signals to which they are exposed. For example, DAPk induces p53 activation in an apoptotic checkpoint, which occurs under oncogenic stress and initiates caspase-dependent type-I cell death. ${ }^{4}$ In contrast, TGF- $\beta$-induced type-I cell death is mediated by DAPk in a p53-independent manner. ${ }^{5}$ Another mode of programmed cell death in which DAPk is involved in some cellular settings is autophagic type-II cell death, characterized by the formation of double-membrane vesicles that consume cell contents. ${ }^{6}$ Overexpression of DAPk leads, in some cells, to the appearance of autophagic vesicles and cell death that does not involve activation of caspases. ${ }^{7}$ However, though it is associated with a large number of cell death scenarios, relatively few specific downstream targets of DAPk, such as direct substrates and interacting proteins, have been identified and the molecular mechanisms underlying DAPk's function are still not well defined. Also, a possible link to type-III necrotic cell death has not been studied so far.

Oxidative stress imposes severe damage to cellular components leading to necrotic type of cell death and is highly associated with the progression of age-related diseases and cancer. ${ }^{8-10} \mathrm{C}-\mathrm{Jun}-\mathrm{N}$-terminal kinase (JNK) is a key node of the cell death network activated under oxidative stress. $^{9,11,12}$ Yet, delineation of the upstream components regulating JNK activation, which eventually dictate cell fate, remains lacking. Protein kinase D 1 (PKD 1, also known as PKC $-\mu),{ }^{13,14}$ a kinase classified within the CAM kinase family, has been identified as one of the upstream regulators of this pathway, required for JNK activation and induction of cell death under oxidative stress conditions. ${ }^{15}$ In general, two modes of PKD activation have been reported to function under oxidative stress, one depending on PKC activation ${ }^{16,17}$ and the second, shown to be wired to the induction of JNK activity through ASK1, being PKC-independent. ${ }^{15,18}$ Yet, the mechanism of the latter PKC-independent activation of PKD, initiating the induction of JNK signaling, remains undefined. In this study, we examined a role for DAPk in response to oxidative stress. We show that DAPk is a regulator of PKD signaling and is required for JNK activation under oxidative stress. We thus gain insight into both the downstream targets of DAPk as well as the components regulating the oxidative stress-responsive pathway.

\section{Results}

DAPk interacts with PKD in an oxidative stressregulated manner. In co-immunoprecipitation studies performed in 293T cells expressing FLAG-DAPk and PKD-

\footnotetext{
${ }^{1}$ Department of Molecular Genetics, Weizmann Institute of Science, Rehovot 76100, Israel

*Corresponding author: A Kimchi, Department of Molecular Genetics, Weizmann Institute of Science, Rehovot 76100, Israel.Tel: +972 89342428 ;

Fax: + 9728 9315938; E-mail: adi.kimchi@ weizmann.ac.il

Keywords: DAPk; PKD; JNK; oxidative stress

Abbreviations: DAPk, DAP kinase; JNK, c-Jun-N-terminal kinase; PKD, protein kinase D

Received 18.12.06; revised 25.6.07; accepted 17.7.07; Edited by P Vandenabeele; published online 17.8.07
} 
GFP, we found that PKD was specifically pulled down by DAPk (Figure 1a). This interaction was verified in a reciprocal experiment in which DAPk was found to co-immunoprecipitate with PKD (Figure 1b). In addition, we observed co-immunoprecipitation of the endogenous proteins. Endogenous PKD was co-immunoprecipitated with endogenous DAPk (Figure 1ci), and in the reciprocal experiment endogenous DAPk was found to coimmunoprecipitate with endogenous PKD (Figure 1cii), indicating that the two kinases interact in vivo. Multiple lines of evidence suggest PKD as a sensor of oxidative stress involved in several signaling pathways. ${ }^{15-17}$ Therefore, we asked whether the interaction of PKD with DAPk might be regulated by oxidative stress. To induce oxidative stress, cells were treated with hydrogen peroxide $\left(\mathrm{H}_{2} \mathrm{O}_{2}\right)$ for $2 \mathrm{~min}$. Notably, hydrogen peroxide treatment enhanced the interaction between PKD and DAPk, as evident by increased levels of co-immunoprecipitation of PKD with DAPk and vice versa (Figure 1a-ci and ii), demonstrating that this association is subjected to regulation by oxidative stress.

PKD is a substrate of DAPk. To determine whether DAPk phosphorylates PKD, an in vitro kinase assay was performed. Immunoprecipitated FLAG-DAPk, or a recombinant fragment of the DAPk catalytic domain only
(DK1), were incubated in a kinase reaction mixture with PKD-GFP. PKD was heat-inactivated before the reaction to avoid autophosphorylation (left lanes of Figure $2 a$ and b). This heat-inactivated form of PKD was specifically phosphorylated both by the catalytic domain of DAPk, as well as by the full-length kinase (Figure $2 a$ and $b$ ), indicating that PKD is a substrate of DAPk.

DAPk induces PKD activation. That PKD is an interacting partner and a substrate of DAPk suggests a cascade of events in which DAPk acts upstream to PKD. A possible role for DAPk in PKD activation was thus examined. To evaluate the effect of DAPk on PKD's kinase activity, a substrate phosphorylation assay was performed. Following incubation of PKD with either DAPk or vehicle in a kinase reaction containing non-labeled ATP, a peptide phosphorylation assay was performed in which syntide-2 was phosphorylated by $\mathrm{PKD}$ in the presence of ${ }^{33} \mathrm{P}$-labeled $\gamma$-ATP. Substrate phosphorylation was then measured by scintillation counting. Pre-incubation of PKD with DAPk resulted in a $30 \%$ increase in PKD's kinase activity as measured by direct phosphotransfer to syntide-2 (Figure 2c). Of note, since the use of purified proteins in vitro is limiting and can reflect only direct conformational changes resulting from the phosphorylation itself, it may be possible that

a

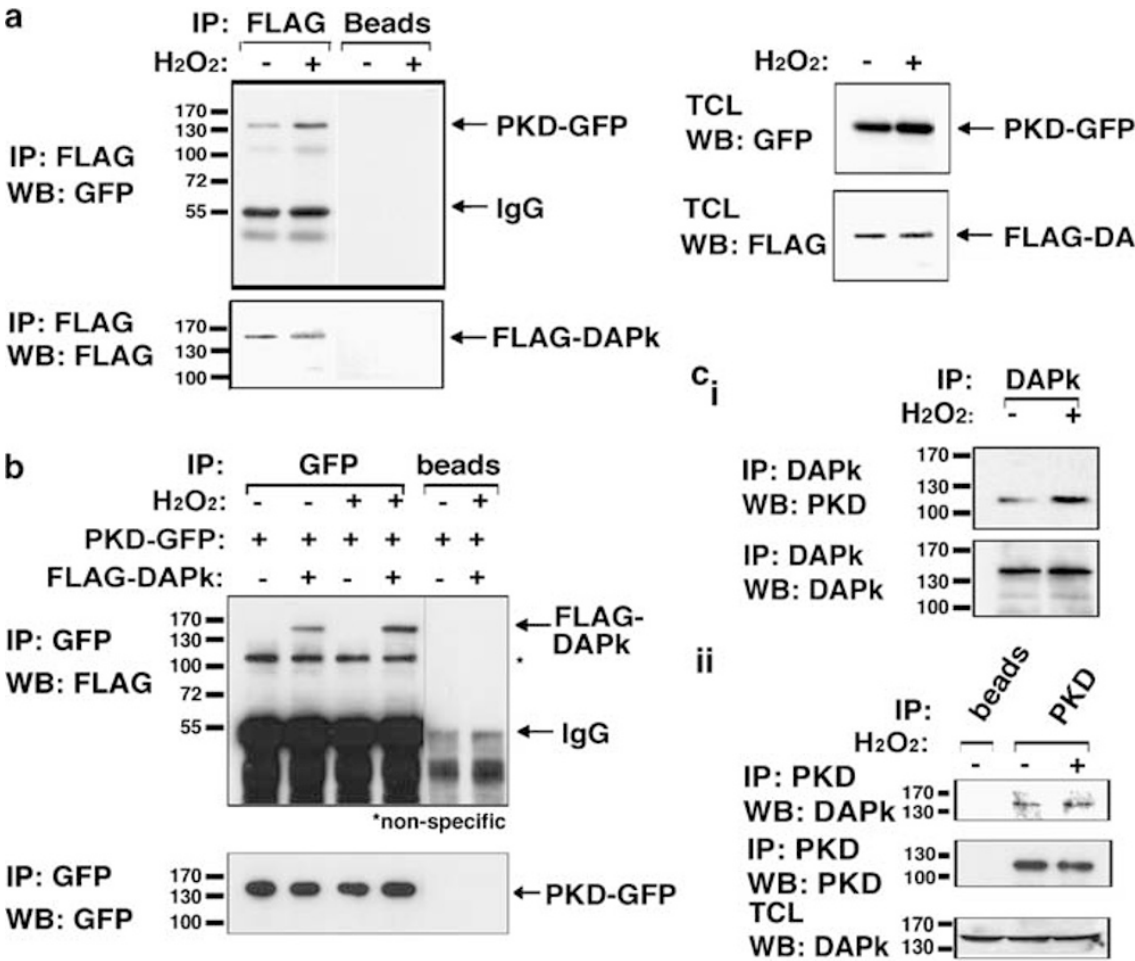

Figure 1 PKD interacts with DAPk in an oxidative stress-regulated manner. (a and $\mathbf{b})$ FLAG-DAPk and PKD-GFP were co-expressed in 293T cells. FLAG-DAPk (a) or PKD-GFP (b) was immunoprecipitated by antibodies against either FLAG or GFP, respectively, from cells that were either treated with $0.5 \mathrm{mM} \mathrm{H}_{2} \mathrm{O}_{2}$ for 2 min or left untreated. Empty beads were used as control for nonspecific binding. Co-immunoprecipitation was observed by Western blot analysis with the indicated antibodies. Expression levels are shown in samples from the total cell lysate (TCL). *a band that was verified as nonspecific in control experiments. (c) (i) Endogenous DAPk was immunoprecipitated from 293T cells that were either treated with $0.5 \mathrm{mM} \mathrm{H}_{2} \mathrm{O}_{2}$ for 5 min or left untreated. Membranes were blotted with anti-PKD or anti-DAPk antibodies as indicated. (ii) Endogenous PKD was immunoprecipitated from 293T cells that were either treated with $0.5 \mathrm{mM} \mathrm{H}_{2} \mathrm{O}_{2}$ for 5 min or left untreated. Membranes were blotted with anti-PKD or anti-DAPk antibodies as indicated. Empty beads were used as control for nonspecific binding 

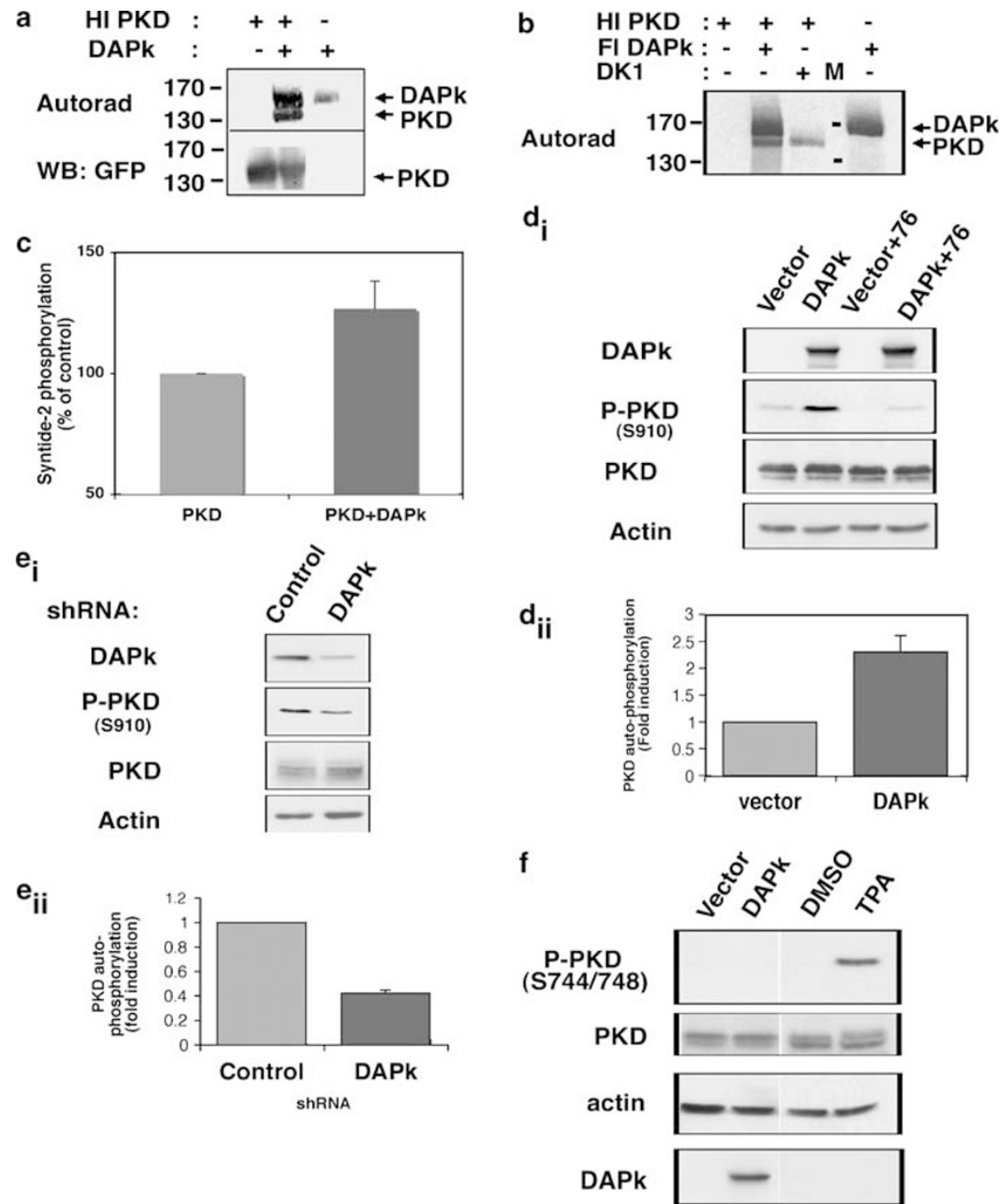

Figure 2 Phosphorylation and activation of PKD by DAPk. (a) Immunoprecipitated PKD-GFP was heat-inactivated (HI) for 15 min at $50^{\circ} \mathrm{C}$ to avoid autophosphorylation and incubated with immunoprecipitated FLAG-DAPk, as indicated, in a kinase reaction mixture containing ${ }^{33}$ P-labeled $\gamma$-ATP. Top: exposure to X-ray film (autorad); bottom: blotting of the same membrane with antibodies against GFP. (b) Full-length (FI) or a recombinant fragment of the catalytic domain only (DK1) of DAPk were incubated with heat-inactivated (HI) PKD-GFP and the kinase reaction was carried out as in (a). (c) Purified PKD was pre-incubated with either DAPk (PKD + DAPk) or control buffer (PKD) in a kinase reaction containing non-labeled ATP, and then transferred to a kinase reaction containing syntide-2 and ${ }^{33}$ P-labeled $\gamma$-ATP. Syntide-2 phosphorylation was measured by scintillation counting. (d) 293T cells were transfected with either empty or FLAG-DAPk expression vectors. $20 \mathrm{~h}$ post transfection the cells were treated with $0.5 \mathrm{mM} \mathrm{H}_{2} \mathrm{O}_{2}$ for two minutes. Where indicated, cells were incubated with Gö6976 (marked as 76) $(1 \mu \mathrm{M})$ for 60 min before treatment. (i) Membranes were blotted with the indicated antibodies. (ii) Statistical analysis of at least three independent experiments. (e) 293T cells were transfected with either DAPk-targeting or control (HcRed-targeting) shRNA plasmids. Seventy-two hours after shRNA transfection, the cells were treated with $0.5 \mathrm{mM} \mathrm{H}_{2} \mathrm{O}_{2}$ for $2 \mathrm{~min}$. (i) Membranes were blotted with the indicated antibodies. (ii) Statistical analysis of at least three independent experiments. (f) $293 T$ cells were transfected either with empty or FLAG-DAPk expression vectors, as control cells were treated with either DMSO or TPA (100 nM). Membranes were blotted with the indicated antibodies

activation of PKD by DAPk occurs to even a larger extent in vivo and may require additional components that are absent in the test tube. Control reactions of PKD with no syntide-2, DAPk incubated with syntide-2 or syntide-2 alone did not produce scintillation counting above background (data not shown).

Autophosphorylation of PKD on serine 910 reflects the status of its catalytic activity and is often used to determine PKD activation. ${ }^{19}$ To further examine a role for DAPk in PKD activation, the effect of DAPk on PKD autophosphorylation levels was evaluated. Autophosphorylation of PKD at serine 910 was enhanced upon overexpression of DAPk by an average of 2.3-fold (Figure 2di and ii). As control, treatment of cells with Gö6976, a PKD inhibitor, blocked the DAPk-induced PKD autophosphorylation, indicating that phosphorylation at position 910 indeed requires the catalytic activity of the kinase (Figure 2di). We next examined whether DAPk was required for PKD activation under oxidative stress by using RNA 
interference against DAPk. Knockdown of DAPk repressed PKD activation as evident by a reduction of 2.5 -fold (in average) in PKD autophosphorylation (Figure 2ei and ii). In addition, expression of a kinase-dead mutant of DAPk also inhibited PKD autophosphorylation (Figure 3a). Taken together, these findings suggest that DAPk is a regulator of PKD activation.

DAPk does not phosphorylate the PKD activation loop. It has been shown that members of the PKC family that act as upstream kinases of PKD phosphorylate the PKD activation loop (serines 744/748). ${ }^{16}$ We thus examined whether DAPk can also induce phosphorylation of these residues by using phospho-specific antibodies against these sites. As control, cells were treated with TPA, an activator of $\mathrm{PKC}$ signaling, that induces the phosphorylation of the PKD activation loop. While TPA treatment resulted in PKD activation loop phosphorylation, DAPk expression did not have any effect (Figure 2f). This suggests that in contrast to members of the PKC family, DAPk can lead to PKD activation and autophosphorylation but does not phosphorylate PKD on serines 744/748 in the activation loop.

DAPk is a mediator of JNK signaling. Activation of JNK signaling is a prominent cellular response to oxidative stress. Our finding that DAPk is part of the oxidative stress-regulated cellular machinery led us to ask whether DAPk could act as a regulator of JNK signaling. To investigate this matter, cells transiently overexpressing DAPk were treated with hydrogen peroxide, and JNK phosphorylation was evaluated in comparison to cells transfected with empty vector only. Remarkably, ectopic expression of DAPk led to a marked increase in JNK phosphorylation (Figure 3a). Statistical analysis revealed that JNK phosphorylation increased by 3.2-fold (in average) upon ectopic expression of DAPk. The kinase-dead mutant of DAPk did not elevate JNK phosphorylation, indicating that the catalytic activity of DAPk is critical for the induction of JNK phosphorylation (Figure 3a). Moreover, the kinase-dead version of DAPk had an inhibitory effect on the endogenous proteins and its expression inhibited JNK activation as evident by a $40 \%$ reduction (in average) in JNK phosphorylation (Figure 3a). Furthermore, in cells in which DAPk expression was knocked down, JNK activation in response to oxidative stress was reduced by $50 \%$ in average (Figure $3 \mathrm{~b}$ ). These data demonstrate that DAPk is a positive mediator of JNK signaling and is indispensable for JNK activation under oxidative stress. The fact that the catalytic activity of DAPk is required for this function suggests that it is through phosphorylation of downstream targets that DAPk elicits the induction of JNK signaling.

DAPK regulates JNK activation via PKD. The finding that DAPk is necessary for the activation of JNK under oxidative stress, a process in which PKD has recently been shown to play an important role, ${ }^{15}$ and that the association of DAPk and $\mathrm{PKD}$ is regulated by oxidative stress, raised the possibility that the DAPk-induced JNK activation may be mediated by PKD. Therefore, DAPk-induced JNK activation was assessed in DAPk overexpressing cells in which PKD activity was inhibited. Two classes of cell permeable PKC family inhibitors were used. The first, Gö6976, inhibits classical PKC isoforms as well as PKD. The second, Gö6983, inhibits both classical and novel PKC isoforms, but does not inhibit PKD, and can thus be used to differentiate between PKD- and PKC-dependent processes. $^{20}$ Inhibition of PKD by treatment of cells with Gö6976 blocked the DAPk-induced JNK phosphorylation
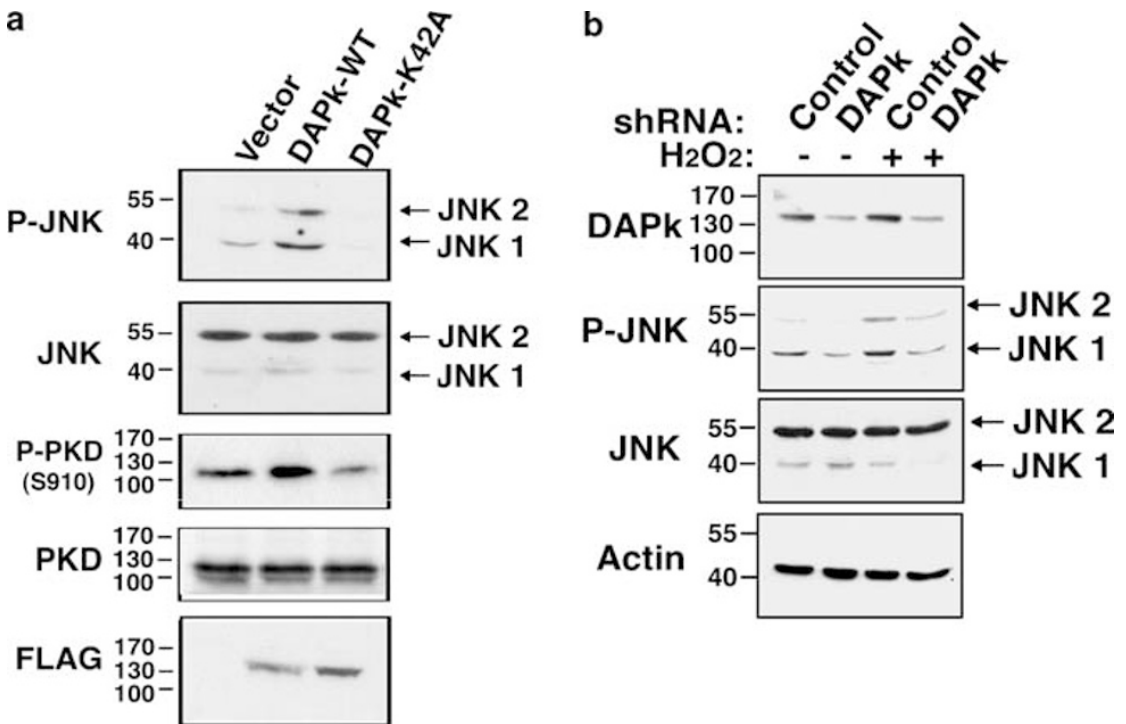

Figure 3 DAPk induces JNK signaling. (a) 293T cells ectopically expressing either empty vector or the wild-type (WT) or kinase-dead versions (K42A) of FLAG-DAPk were treated with $0.5 \mathrm{mM} \mathrm{H}_{2} \mathrm{O}_{2}$ for $2 \mathrm{~min}$. Membranes were blotted with the indicated antibodies. (b) 293T cells were transfected with either DAPk-targeting or control (HcRedtargeting) shRNA plasmids. Seventy-two hours after transfection, the cells were either treated with $0.5 \mathrm{mM} \mathrm{H}_{2} \mathrm{O}_{2}$ for 2 min or left untreated. Membranes were blotted with the indicated antibodies 
(Figure 4a). In contrast, inhibition of other PKC isoforms by Gö6983 had no effect on the DAPk-induced JNK phosphorylation, suggesting that DAPk functions in a PKCindependent manner (Figure 4a). To further examine this finding, we used RNA interference against PKC- $\delta$, a known upstream kinase of PKD. Interestingly, knockdown of PKC- $\delta$ did not effect JNK activation by DAPk, supporting a PKCindependent function of DAPk (Figure 4b). We next examined the DAPk-induced JNK phosphorylation in cells knocked down in PKD expression by RNA interference. As RNA interference might produce off-target effects, we used either siRNA (Figure 4c) or shRNA (Figure 4d) containing different targeting sequences against PKD. In both cases, knockdown of PKD hindered the DAPk-induced JNK phosphorylation, consistent with the effect observed upon PKD inhibition by Gö6976 (Figure 4c and d). In addition, rescue experiments were performed by overexpressing PKD to an extent that exceeded the capacity of the siRNA to reduce effectively the protein levels. Under these conditions, re-introduction of PKD in the presence of the specific siRNA rescued the inhibitory effect of the siRNA on JNK phosphorylation (data not shown). This suggests that PKD is specifically required for JNK activation. These findings signify that DAPK provokes JNK signaling in a PKDdependent manner and suggest a signaling cascade in which under oxidative stress, DAPk activates PKD leading to the induction of JNK phosphorylation.

DAPk is indispensable for cell death under oxidative stress. Tumor suppressor proteins, such as DAPk, are crucial for the correct execution of cell death under stress conditions. A direct involvement of DAPK in oxidative stressinduced cell death was not studied yet, and questions such as whether DAPk is being activated in this cellular setting were not addressed as well. Under basal conditions, DAPk is regulated by an autoinhibitory phosphorylation of serine 308 (S308), which locks its catalytic cleft thus rendering the kinase inactive. ${ }^{21}$ Activation of DAPk has been shown to involve the dephosphorylation of $\mathrm{S} 308$ by an unknown phosphatase. ${ }^{21}$ We thus investigated the activation of DAPk under oxidative stress by immunoprecipitation of the phospho-S308-positive DAPk molecules under basal or stressed conditions. DAPk dephosphorylation was observed shortly after addition of hydrogen peroxide to the cells as evident by reduced levels of DAPk, which were immunoprecipitated by the antiphospho-S308 antibody, whereas the cellular level of total DAPk remained unchanged (Figure 5a). This suggests that DAPk is activated as an early step in response to oxidative stress.

Finally, cell death was considered to evaluate the contribution of DAPk to the oxidative stress response. To this end, cells in which DAPk expression was knocked down, or control cells, were incubated with hydrogen peroxide for $16 \mathrm{~h}$ and cell death was measured by counting trypan bluepositive cells. The reduction in DAPk expression levels in the knocked down cells is demonstrated in a representative Western blot analysis (Figure 5b). Cells knocked down in DAPk expression failed to execute the full-cell death responses to oxidative stress as compared to the responses of control cells with unperturbed DAPk expression (Figure $5 \mathrm{c}$ ) and displayed a lower fraction of trypan blue-positive cells (Figure $5 d$ ). This indicates that DAPk is indispensable for programmed cell death under oxidative stress. Interestingly, the observed cell death was caspase-independent as no caspase-3 cleavage was observed in hydrogen peroxidetreated cells (Figure 5ei). Caspase cleavage was observed in
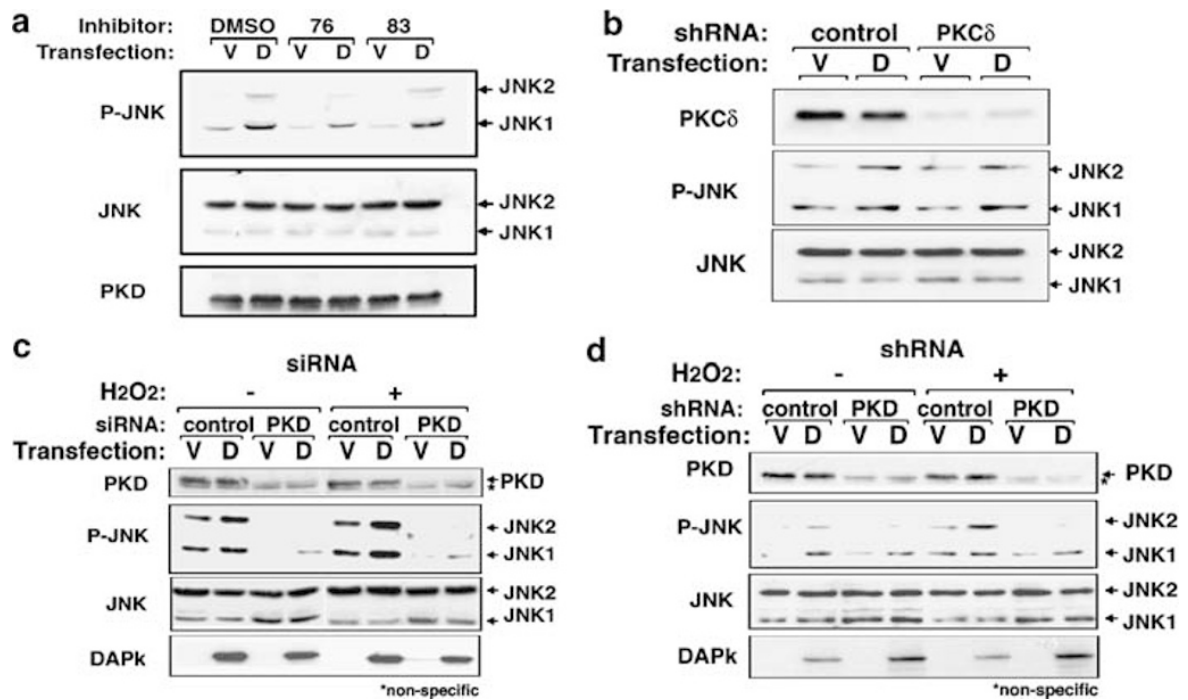

Figure 4 DAPk-induced JNK activation is mediated by PKD. (a) 293T cells ectopically expressing either DAPk (D) or empty vector (V) were incubated with either Gö6976 $(1 \mu \mathrm{M})$, Gö6983 $(1 \mu \mathrm{M})$ or DMSO as control for 60 min and then treated with $0.5 \mathrm{mM} \mathrm{H}_{2} \mathrm{O}_{2}$ for 2 min. Membranes were blotted with the indicated antibodies. (b) PKC- $\delta$ targeting or control shRNA plasmids were transfected into 293T cells. Forty-eight hours after transfection, the cells were transfected again with either DAPk (D) or empty vector (V) for $24 \mathrm{~h}$. Membranes were blotted with the indicated antibodies. (c) PKD-targeting or control siRNA were transfected into 293T cells. Forty-eight hours after transfection, the cells were transfected again with either DAPk (D) or empty vector (V). The following day the cells were either treated with $0.5 \mathrm{mM} \mathrm{H}_{2} \mathrm{O}_{2}$ for 2 min or left untreated, and the membranes were blotted with the indicated antibodies. (d) PKD-targeting shRNA was used as in (c) 
a

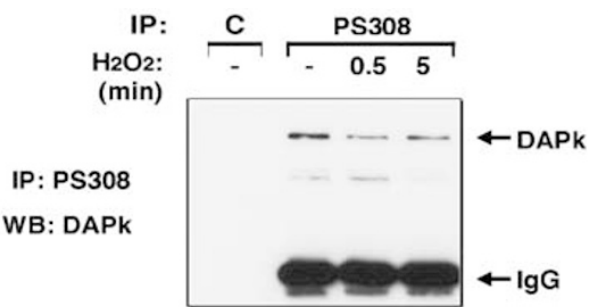

TCL

WB: DAPk

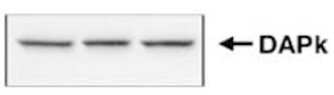

b

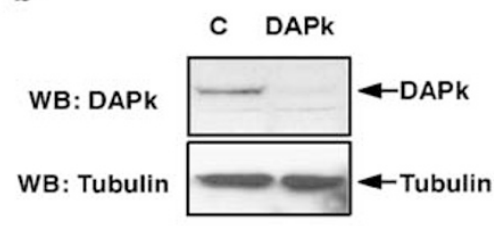

C

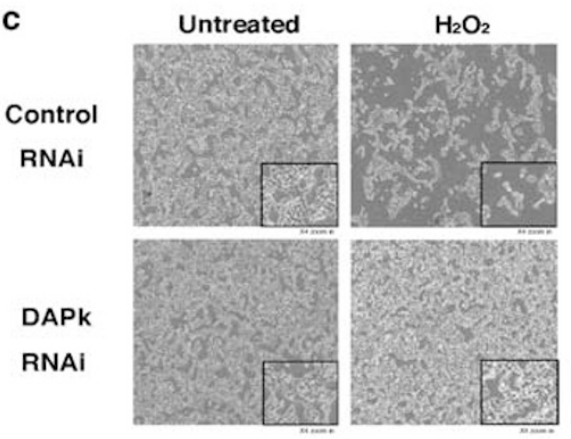

$\mathbf{e}_{\mathbf{i}}$

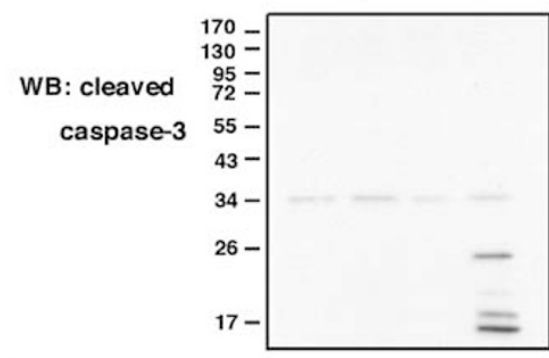

ii

WB: HMGB1 d
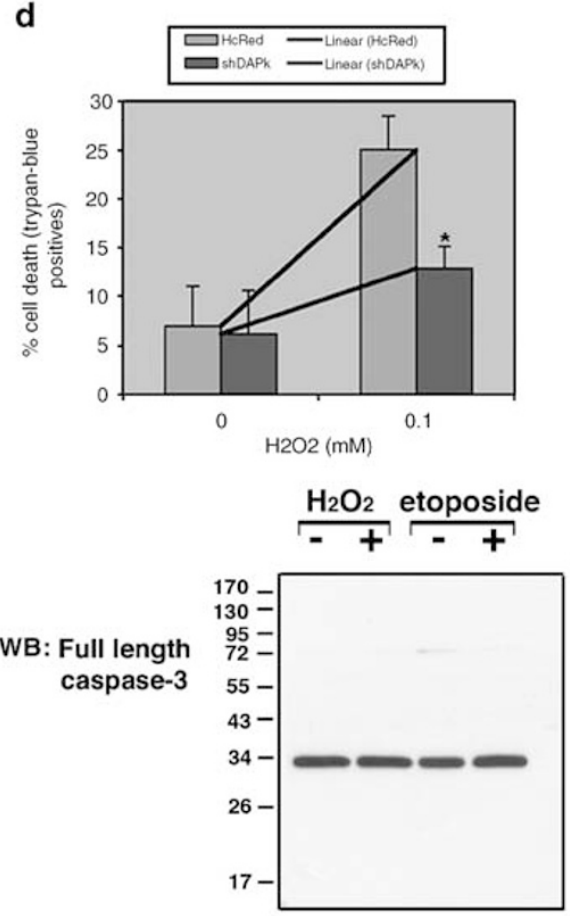

Figure 5 DAPk is activated under oxidative stress and is indispensable for cell death. (a) Endogenous DAPk phosphorylated on S308 was immunoprecipitated by antiphospho $\mathrm{S} 308$ antibodies following $\mathrm{H}_{2} \mathrm{O}_{2}$ treatment for the indicated times. $\mathrm{C}=$ control immunoprecipitation with empty beads. Western blot analysis of the total cell lysate (TCL) with anti-DAPk is shown for total expression levels. (b-d) 293T cells transfected with either DAPk-targeting or control (HcRed-targeting) shRNA plasmids were treated with $0.1 \mathrm{mM} \mathrm{H}_{2} \mathrm{O}_{2}$ for $16 \mathrm{~h}$ or left untreated. (b) A representative Western blot analysis of the control (marked C) and DAPk RNAi transfected cells. (c) Following $\mathrm{H}_{2} \mathrm{O}_{2}$ treatment, the cells were visualized by light microscopy. (d) Cell death was measured by counting trypan blue-positive cells out of the total cell number. For each population, at least 150 cells, from each of three individual plates, were counted. Data represent means of four individual experiment \pm S.D. Statistical analysis was performed by a two-tailed Student's $t$-test. ${ }^{\star} P=0.007$. (e) $293 \mathrm{~T}$ cells were treated with $0.1 \mathrm{mM} \mathrm{H}_{2} \mathrm{O}_{2}$ for $16 \mathrm{~h}$ or left untreated. (i) As a positive control cells were treated with $50 \mu \mathrm{M}$ etoposide. Membranes were blotted with antibodies against full-length or the cleaved form of caspase-3. (ii) Samples from the media were collected and analyzed for release of HMGB1 by blotting with specific antibodies

cells treated with etoposide, an agent known to induce type-I cell death (Figure 5ei), indicating that though caspases can be activated in these cells, oxidative stress did not induce type-I cell death. Reactive oxygen species, such as hydrogen peroxide, often induce necrotic cell death. HMGB1, a nuclear protein that is released from the cell to the extracellular milieu during necrosis, was detected in the media of hydrogen peroxide-treated cells (Figure 5eii). This suggests that DAPk is required for oxidative stress-induced necrotic cell death.

\section{Discussion}

We have previously reported the cloning of DAPk as a positive mediator of cell death and characterized its tumor-suppressive functions. ${ }^{1-4}$ Though a large number of physiological examples in which DAPk is pivotal for programmed cell death has accumulated over the years, only a few specific activating signals as well as relevant substrates and interacting proteins have been described. In this work, we demonstrate DAPk as a sensor of oxidative stress and describe PKD as a novel target 
of DAPk that mediates JNK activation and caspase-independent cell death under oxidative stress.

Different groups have previously reported the involvement of PKD in oxidative stress-regulated signaling events. Interestingly, PKD was described as a regulator of either cell survival or cell death, reflecting the complexity of molecular networks, in general, in which a central node such as PKD can shift between a pro-survival and pro-death function depending upon the status of other genes in the network. Work from the laboratories of Toker and Rozengurt described Src and PKC- $\delta$ as modulators of PKD activity under oxidative stress. Phosphorylation of tyrosine 463 in PKD by Src, in concert with phosphorylation of the PKD activation loop by PKC- $\delta$, were shown to induce the activation of NF- $\kappa$ B by PKD leading to increased cellular survival. ${ }^{16,17}$ More recently, Zhang et al. ${ }^{15}$ demonstrated a PKC-independent activation mode of PKD under oxidative stress, which stimulates JNK phosphorylation via the activation of ASK1, consequently leading to cell death. However, how PKD is regulated in the latter cellular setting was not described. Our results suggest DAPk as one possible missing link in this pathway. By knocking down DAPk expression in cells, we have demonstrated that DAPk is an important factor for the activation of JNK under oxidative stress. Moreover, the induction of JNK activity by DAPk was wired through PKD, as inhibition of PKD by a chemical inhibitor or knockdown of PKD expression by different RNAi sequences abrogated the DAPk-induced JNK phosphorylation. Of note, inhibition of PKC's did not have the same effect on DAPk, nor did DAPk induce PKD activation loop phosphorylation, suggesting that this specific signaling cascade is indeed PKC-independent as reported by Zhang et al. ${ }^{15}$ This indicates that DAPK stimulates JNK activation under oxidative stress in a PKD-dependent manner. The observed reduction in the autophosphorylation level of PKD in DAPk knocked down cells, taken together with the finding that DAPk binds PKD and that this binding increases in response to oxidative stress, implicate DAPk in the activation of PKD and depict DAPk as an upstream activator of PKD under oxidative stress. Nevertheless, while the reduction in JNK phosphorylation upon knockdown of PKD was almost complete, knockdown of DAPk resulted in only a partial effect on JNK phosphorylation. Although different proteins may be influenced differently by the extent of their knockdown, these observations may suggest the existence of yet another pathway for JNK activation by PKD, which is DAPkindependent. Since no connectivity of the DAPk-dependent pathway to the PKC-dependent pathway could be identified, it is plausible that these represent two parallel modes for PKDinduced JNK activation.

Finally, a requirement of DAPk for cell death under oxidative stress, shown here by the increased resistance of the DAPk knocked down cells to the stress, suggests that activation of PKD by DAPk shifts its function toward promotion of cell death. This is in accord with the known role of DAPk as a tumor suppressor and as a positive mediator of cell death.

Oxidative stress has been linked to the progression of several human diseases including cancer. As oxidation may cause irreversible damage to cellular contents, a signaling network that includes both pro-survival and pro-death functional arms is initiated upon exposure to oxidizing agents. The correct balance between activation of these arms is critical for healthy growing cells. The observation made here, that DAPk is activated by dephosphorylation of $\$ 308$ shortly after exposure to oxidative stress, suggests a model in which DAPk acts as an upstream regulator in this signaling network. To date, a specific phosphatase for S308 in DAPk has not been identified and further studies may be required to explore the mechanism of DAPk dephosphorylation. Results presented in this work imply that early events initiated by DAPk are required for the sensitization of cells to oxidative stress and for the execution of cell death. It is thus plausible that loss of DAPk will render the cells susceptible to continuous growth with damaged cellular contents, a condition that may eventually progress to disease. While no caspase activation was detected in cells after treatment of hydrogen peroxide, we observed the release of HMGB1 to the extracellular milieu. Our data thus concur with the fact that cell death initiated by oxidative stress is commonly characterized as necrosis and establish for the first time a novel molecular pathway emerging from DAPk, which culminates in type-III necrotic cell death.

In conclusion, this work maps DAPk within the oxidative stress signaling pathway in a position that serves to activate $\mathrm{PKD}$, here identified as a substrate of DAPk. By revealing a novel mechanism of JNK activation via DAPk, these findings provide an advance in both the field of DAPk as well as in the understanding of the molecular events taking place during the cellular response to oxidative stress, which culminate in caspase-independent necrotic cell death.

\section{Materials and Methods}

Cell lines and transfections. HEK 293T cells were grown in Dulbecco's modified Eagle's medium (Biological Industries, Beit Haemek, Israel) supplemented with $10 \%$ fetal bovine serum. For transient transfections, cells were grown to approximately $60 \%$ confluence, and transfections were performed by the calcium phosphate method.

DNA constructs. FLAG-tagged wild-type or kinase-dead (K42A) DAPk constructs were described previously. ${ }^{22,23}$ GFP-tagged PKD1 was kindly provided by Pfizenmaier K (Stuttgart, Germany).

RNA interference. DAPk expression was knocked down by the targeting sequence CATGGAGAAATTCAAGAAG corresponding to nucleotides 1210-1228 of human DAPk (NM_004938) inserted into pSUPER. As shRNA control, the nonmammalian protein HcRed (AF363776) was targeted by the sequence GTATGC GCATCAAGATGTA corresponding to nucleotides 99-117. PKD1 expression was knocked down either by PKD-targeting shRNA in pSUPER vector kindly provided by Dr Alex Toker (Harvard Medical School, Boston, MA) or by siGENOME SMARTpool siRNA reagent against human PRKCM (NM_002742; Dharmacon, CO, USA). For control siRNA, siCONTROL non-targeting siRNA\#1 was used (Dharmacon). PKC- $\delta$ shRNA in pSUPER vector was kindly provided by Dr Alex Toker.

Cell extracts and immunoprecipitation. Cells were lysed in cold $A$ buffer $^{24}$ for ectopically expressed proteins, or in RIPA buffer $(50 \mathrm{mM}$ Tris $\mathrm{pH} 7.4$, $150 \mathrm{mM} \mathrm{NaCl}, 1 \mathrm{mM}$ EDTA, $1 \%$ Triton $\mathrm{X}-100,1 \%$ sodium deoxycholate, $0.1 \%$ SDS) for the endogenous proteins. Following pre-clearance in protein G PLUS-Agarose (Santa Cruz Biotechnology, CA, USA), the extracts were incubated with either antiFLAG, anti-GFP, anti-DAPk or anti-PKD antibodies for $90 \mathrm{~min}$. Following repeated washing, the immunocomplexes were boiled in $\times 1$ sample buffer, separated on SDS-PAGE and subjected to immunoblotting.

Antibodies and reagents. Agarose-conjugated anti-FLAG M2 gel beads, anti-actin AC-40, anti- $\beta$-tubulin, anti-DAPk, antiphospho S308-DAPk, FLAG peptide, bovine calmodulin, etoposide and hydrogen peroxide (Sigma-Aldrich, 
MO, USA); anti-GFP clones 7.1 and 13.1 (Roche Applied Science, IN, USA); antiphospho-JNK G9 (Thr183 and Tyr185), anti-JNK, antiphospho-PKD (Ser910), antiphospho-PKD (Ser744/748) and anti-cleaved caspase-3 (Cell Signaling Technology, MA, USA); anti-PKD D-20, anti-caspase-3 and anti-PKC- $\delta$ (Santa Cruz Biotechnology); anti-HMG1 (BD Pharmingen, CA, USA); and PKC inhibitors Gö6976, Gö6983 (Calbiochem, CA, USA).

In vitro kinase assay. 293T cells were transfected with FLAG-DAPK or PKDGFP constructs. Cells were lysed and proteins were immunoprecipitated and/or eluted as follows: for FLAG immunoprecipitation, cells were lysed in cold B buffer. ${ }^{23}$ Following pre-clearance of the extracts with protein G PLUS-Agarose for $30 \mathrm{~min}$ on rotation at $4^{\circ} \mathrm{C}$, cleared extracts were incubated with agarose-conjugated anti-FLAG M2 gel beads for $90 \mathrm{~min}$ at $4^{\circ} \mathrm{C}$. Immunoprecipitates were washed extensively with $B$ buffer and two times with $54 \mathrm{~K}$ buffer ${ }^{23}$ The proteins were eluted by the addition of $50 \mu \mathrm{g}$ of FLAG peptide. For GFP immunoprecipitation, cells were lysed in cold RIPA buffer. Extracts were pre-cleared as described above, incubated with $4 \mu \mathrm{g}$ anti-GFP antibodies overnight at $4^{\circ} \mathrm{C}$ and then protein A/G PLUS-Agarose was added for 90 min. Immunoprecipitates were washed extensively in RIPA buffer. Immunoprecipitated FLAG-DAPk was quantified after elution against standards. PKD-GFP was heat-inactivated $\left(15 \mathrm{~min}, 50^{\circ} \mathrm{C}\right)$ and incubated with either full-length FLAG-DAPk or a recombinant fragment of the DAPk catalytic domain (DK1) in DAPk reaction buffer $(50 \mathrm{mM}$ HEPES, $\mathrm{pH} 7.5,20 \mathrm{mM} \mathrm{MgCl}$ ) containing $1 \mu \mathrm{M}$ bovine calmodulin, $0.5 \mathrm{mM} \mathrm{CaCl} 2,10 \mu \mathrm{Ci}$ of ${ }^{33} \mathrm{P}$ labeled $\gamma$-ATP and $50 \mu \mathrm{M}$ ATP. The kinase assay was conducted at $30^{\circ} \mathrm{C}$. Protein sample buffer was added to terminate the reaction, and after boiling, the proteins were analyzed on SDS-PAGE. The gels were either dried or transferred onto nitrocellulose membranes and visualized by autoradiography or immunoblotting.

Peptide phosphorylation. PKD immune complexes were incubated with either purified DAPk or vehicle in DAPk-reaction buffer containing only non-labeled ATP for $20 \mathrm{~min}$ at $30^{\circ} \mathrm{C}$. The immune complexes were then washed in PKD-reaction buffer (30 mM Tris- $\mathrm{HCl}, \mathrm{pH} 7.4,10 \mathrm{mM} \mathrm{MgCl}, 2 \mathrm{mM}$ dithiothreitol), and substrate peptides (syntide-2, $2 \mu \mathrm{g}$; Calbiochem) were added in the presence of ${ }^{33} \mathrm{P}$-labeled $\gamma$-ATP and $50 \mu \mathrm{M}$ ATP in PKD-reaction buffer (final reaction volume $30 \mu \mathrm{l}$ ) for $6 \mathrm{~min}$ at $30^{\circ} \mathrm{C}$. Reactions were terminated by spotting onto Whatman $\mathrm{P}-81$ phosphocellulose paper. Papers were washed thoroughly in $\mathrm{H}_{3} \mathrm{PO}_{4}$ and dried, and radioactivity incorporated into peptides was determined by detection of Cerenkov radiation in a scintillation counter.

Cell death. Cell death was measured by trypan blue staining. Cells were collected, stained with trypan blue and counted. In each experiment, approximately 150 cells from each of three individual plates were counted, in duplicates, and averaged. Data were subjected to statistical analysis.

Statistical analysis. Data are presented as means \pm S.D. from at least three experiments. Analysis of densitometry was performed using NIH Image 1.60. Results were normalized to control expression levels. Differences were analyzed by unpaired two-tailed Student's $t$-test. $P<0.05$ was considered significant.

Acknowledgements. We thank $K$ Pfizenmaier for providing the PKD-GFP construct; A Toker for providing the PKD and PKC- $\delta$ shRNA constructs; $Y$ Shoval and $E$ Zalckvar for preparing the shRNA constructs for DAPk and HcRed, respectively; $Y$ Idelchuk for technical assistance. This work was supported by grants from the European Union (LSHB-CT-2004-511983), the Kahn Center for System Biology and from the Center of Excellence grant from Flight Attendant Medical Research Institute (FAMRI); AK is the incumbent of the Helena Rubinstein Chair of Cancer Research.
1. Deiss LP, Feinstein $E$, Berissi $H$, Cohen $O$, Kimchi A. Identification of a novel serine/ threonine kinase and a novel $15-\mathrm{kD}$ protein as potential mediators of the gamma interferoninduced cell death. Genes Dev 1995; 9: 15-30.

2. Bialik S, Kimchi A. DAP-kinase as a target for drug design in cancer and diseases associated with accelerated cell death. Semin Cancer Biol 2004; 14: 283-294.

3. Bialik S, Kimchi A. The death-associated protein kinases: structure, function, and beyond Annu Rev Biochem 2006; 75: 189-210.

4. Raveh T, Droguett G, Horwitz MS, DePinho RA, Kimchi A. DAP kinase activates a p19ARF/p53-mediated apoptotic checkpoint to suppress oncogenic transformation. Nat Cell Biol 2001; 3: 1-7.

5. Jang $\mathrm{CW}$, Chen $\mathrm{CH}$, Chen CC, Chen JY, Su YH, Chen RH. TGF-beta induces apoptosis through Smad-mediated expression of DAP-kinase. Nat Cell Biol 2002; 4: 51-58.

6. Gozuacik D, Kimchi A. Autophagy as a cell death and tumor suppressor mechanism. Oncogene 2004; 23: 2891-2906.

7. Inbal B, Bialik S, Sabanay I, Shani G, Kimchi A. DAP kinase and DRP-1 mediate membrane blebbing and the formation of autophagic vesicles during programmed cell death. J Cell Biol 2002; 157: 455-468.

8. Suh YA, Arnold RS, Lassegue B, Shi J, Xu X, Sorescu D et al. Cell transformation by the superoxide-generating oxidase Mox1. Nature 1999; 401: 79-82.

9. Finkel T, Holbrook NJ. Oxidants, oxidative stress and the biology of ageing. Nature 2000; 408: 239-247.

10. Hussain SP, Hofseth LJ, Harris CC. Radical causes of cancer. Nat Rev Cancer 2003; 3: 276-285.

11. Sakon S, Xue X, Takekawa M, Sasazuki T, Okazaki T, Kojima Y et al. NF-kappaB inhibits TNF-induced accumulation of ROS that mediate prolonged MAPK activation and necrotic cell death. EMBO J 2003; 22: 3898-3909.

12. Kamata $H$, Honda S, Maeda S, Chang L, Hirata $H$, Karin M. Reactive oxygen species promote TNFalpha-induced death and sustained JNK activation by inhibiting MAP kinase phosphatases. Cell 2005; 120: 649-661.

13. Johannes FJ, Prestle J, Eis S, Oberhagemann P, Pfizenmaier K. PKCmu is a novel, atypical member of the protein kinase C family. J Biol Chem 1994; 269: 6140-6148.

14. Valverde AM, Sinnett-Smith J, Van Lint J, Rozengurt E. Molecular cloning and characterization of protein kinase $\mathrm{D}$ : a target for diacylglycerol and phorbol esters with a distinctive catalytic domain. Proc Natl Acad Sci USA 1994; 91: 8572-8576.

15. Zhang W, Zheng $S$, Storz $P$, Min W. Protein kinase $D$ specifically mediates apoptosis signal-regulating kinase 1-JNK signaling induced by $\mathrm{H}_{2} \mathrm{O}_{2}$ but not tumor necrosis factor. J Biol Chem 2005; 280: 19036-19044.

16. Waldron RT, Rozengurt $E$. Oxidative stress induces protein kinase $D$ activation in intact cells. Involvement of Src and dependence on protein kinase C. J Biol Chem 2000; 275: 17114-17121.

17. Stortz $\mathrm{P}$, Doppler $\mathrm{H}$, Toker A. Protein kinase Cdelta selectively regulates protein kinase D-dependent activation of NF-kappaB in oxidative stress signaling. Mol Cell Biol 2004; 24: 2614-2626.

18. Lemonnier J, Ghayor C, Guicheux J, Caverzasio J. Protein kinase C-independent activation of protein kinase $\mathrm{D}$ is involved in BMP-2-induced activation of stress mitogenactivated protein kinases JNK and p38 and osteoblastic cell differentiation. J Biol Chem 2004; 279: 259-264.

19. Matthews SA, Rozengurt E, Cantrell D. Characterization of serine 916 as an in vivo autophosphorylation site for protein kinase D/Protein kinase Cmu. J Biol Chem 1999; 274: 26543-26549.

20. Gschwendt M, Dieterich S, Rennecke J, Kittstein W, Mueller HJ, Johannes FJ. Inhibition of protein kinase $\mathrm{C}$ mu by various inhibitors. Differentiation from protein kinase $\mathrm{c}$ isoenzymes. FEBS Lett 1996; 392: 77-80.

21. Shohat G, Spivak-Kroizman T, Cohen O, Bialik S, Shani G, Berrisi $H$ et al. The proapoptotic function of death-associated protein kinase is controlled by a unique inhibitory autophosphorylation-based mechanism. J Biol Chem 2001; 276: 47460-47467.

22. Cohen O, Feinstein E, Kimchi A. DAP-kinase is a Ca2+/calmodulin-dependent, cytoskeletal-associated protein kinase, with cell death-inducing functions that depend on its catalytic activity. EMBO J 1997; 16: 998-1008.

23. Shani G, Marash L, Gozuacik D, Bialik S, Teitelbaum L, Shohat G et al. Death-associated protein kinase phosphorylates ZIP kinase, forming a unique kinase hierarchy to activate its cell death functions. Mol Cell Biol 2004; 24: 8611-8626.

24. Eisenberg A, Biener E, Charlier M, Krishnan R, Djiane J, Herman B et al. Transactivation of erbB2 by short and long isoforms of leptin receptors. FEBS Lett 2004; 565: 139-142. 